\title{
Research on Innovative Application of Intelligent Chat Robot Based on WebView
}

\author{
Zhu Xiangcai, Zhuo Saifeng, Wang Feifei, and Wang Feng
}

School of Information Science and Technology, Taishan University (TSU), Shandong 271021, China

Keywords: chat robot, artificial intelligence, big data

\begin{abstract}
Artificial intelligence is a hot topic today, and chat robots are among the more sought after research areas. This project briefly introduces the overview of intelligent robots and their research background, and explains the basic principles and key technologies of intelligent robots. Research on big data analysis and deep learning has led to the development of the field of chat robots. In the future, chat robots will gradually replace APP as the next generation service portal of the Internet and will create more room for development. The interface is also different from the chat robots in the past. There is a small robot on the main interface that can naturally communicate with you, close to the user, smart Xiaofeng, which can accurately read out the words you describe, when you communicate with him, he will be very vivid to chat with you, and the chat content will be in the dialogue box appearing in real time.
\end{abstract}

\section{Introduction}

This system is to design a cross-platform intelligent robot based on WebView browser, named "Smart Xiaofeng". Embed WebView in the App's UI for dynamic updates of certain features. The first problem to solve when using Chromium to implement WebView is its dynamic loading problem.

Based on the means of communication and the realization of communication between people, it also makes the communication between people and artificial intelligence system become a reality more quickly. The chat robot is an artificial intelligence system that can communicate online and communicate with people through natural language. The chatbot is essentially an automated question answering system. It is one of the hot topics in computer application research.

The chat robot utilizes natural language processing technology, knowledge base, and real-time updated information resources. On the one hand, it completes the analysis and processing of user problems, and on the other hand, it completes the generation of correct answers. The chat robot system in the prior art generally includes a communication module, a query server, an artificial intelligence server, and a corresponding database. In this chat robot system, a database is used as a carrier of knowledge points, and the user performs various conversations with the chat robot through the instant messaging platform or the short message platform.

\section{Features and Guidance}

Through the recognition of the user's voice, it is translated into text displayed on the message box, and transmitted through the network to form an intelligent robot capable of talking to us (including voice dialogue). The intelligent robot dynamically displays related semantic expressions using relevant character features. We will also judge the user's mood according to the user's tone words, to answer the corresponding speech, intelligent feedback in the background through big data.

The robot is an automated equipment integrating mechanical, electronic, control, computer, sensor, artificial intelligence and other multi-disciplinary advanced technologies, representing the direction of the future development of smart equipment. At present, the state's supportive attitude towards the robot industry is clear, and it gradually assumes a gradual increase.

The future market application of intelligent robots, and the future, the development of intelligent robots will no longer be limited to imitating human conversations, answer awkwardly some basic 
questions, but will be able to truly understand the meaning of dialogues and respond emotionally. The communication application happens to be the latest and most interesting communication method. Today, including the visible future, robots are hardly limited to output content.

Understand the development, classification, and application of intelligent robots and cultivate the interest in learning to use robots. Enhance students' awareness of information and understand the changes in information technology and its impact on work and society.

Understand the basic working principles of intelligent robots and learn the basic knowledge and skills of common sensors and mechanical transmissions.

Learn to cooperate with others and follow the rules of the game while learning and using smart robots. Conditional students can learn the knowledge and skills of simple robot assembly and maintenance.

\section{Research Process}

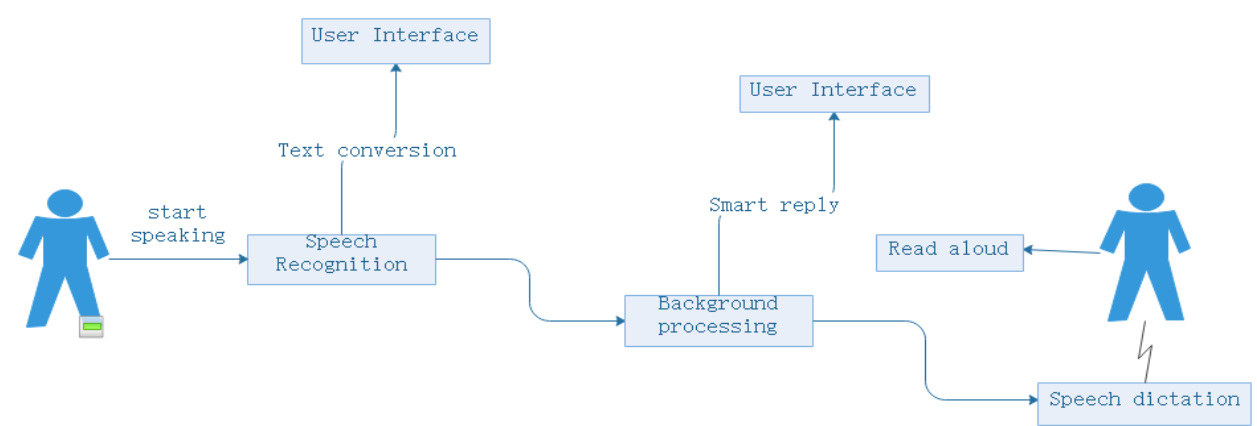

Fig. 1 Software flow chart

The name of this product is Smart Xiaofeng, which can realize an intelligent robot through voice interaction and graphic interaction. The speech interaction at this time can be divided into text form and language form. The language form can be divided into speech feedback and emotion feedback.

Speech recognition is a cross-discipline, and the fields involved are signal processing, pattern recognition, probability theory and information theory, phonation mechanism and auditory mechanism, artificial intelligence, etc. It even involves human body language and behavioral actions can help the other person understand. Its application fields are also very wide. For example, the voice input system relative to the keyboard input method, the voice control system.

Mainly includes the sampling and preprocessing part of speech signal, feature parameter extraction part, speech recognition core part and speech recognition post-processing part. The basic structure of speech recognition system is given in the figure.

Interactive image processing system. It is a relatively convenient and perfect image processing system. With this computer system, the operator and the machine can talk to each other, so the man-machine dialogue processing system. This kind of system has a set of application programs. During the operation process, the computer presents a series of questions to the operator for selection.

The text form means that the user can use the voice recognition to present the user's words in the message box according to the user's spoken text, thereby making the user more convenient. Speech Recognition Search Editing The search in continuous speech recognition is to find a sequence of word models to describe the input speech signal and thus obtain the word decoding sequence. The voice form here refers to our smart Xiaofeng can judge user's mood according to some of the user's mood words to answer according to the user's question.

In the existing speech recognition processing and feedback system, usually include: speech recognition, speech understanding and speech recovery. In the third part of the voice reply module, the use of a single voice tone and no difference reply, basically only divided into male and female voices, the user needs for different voices can only be selected by setting in advance.

Emotion feedback the main function of our smart Xiaofeng is to determine the user's mood based on some user's interjection or other symbols. 


\section{System Test Plan and Test Data}

After smart minipeaks are completed, we will test them. The scheme is as follows:

How many users can it make online at the same time? For our smart Xiaofeng, the number of its users online may allow about 2000 people to use it at the same time, and there will be no stalling.

Perform functional tests on all aspects of its functions to achieve our desired results. The speech recognition function is particularly well implemented and greatly facilitates the use of users. At the same time, the voice feedback and its emotional feedback have also achieved the desired results. According to the user's needs, to make users satisfied with the answer.

The security test has a relatively high security index and brings many benefits. This topic stores the information in the database into knowledge bases, think tanks, event libraries, and action libraries that model the human brain, and queries the database based on the user's vocabulary and sentence types, so that the smart Xiaofeng can more accurate understanding of the user's questions and statements.

The overall effect of testing it is good, and there are a small number of deficiencies we will improve in the later period. The test data is as follows:

First, the dialogue sentences that have appeared on the Internet forums, microblogs, or webpages are extracted and used as training corpus. When the user enters a sentence, it will find a sentence that most closely resembles this sentence, and the answer corresponding to this sentence can be directly output as a reply from the computer. Sometimes, the sentence found by the system may correspond to many replies. It does not know which replies are most suitable for the current input statement.

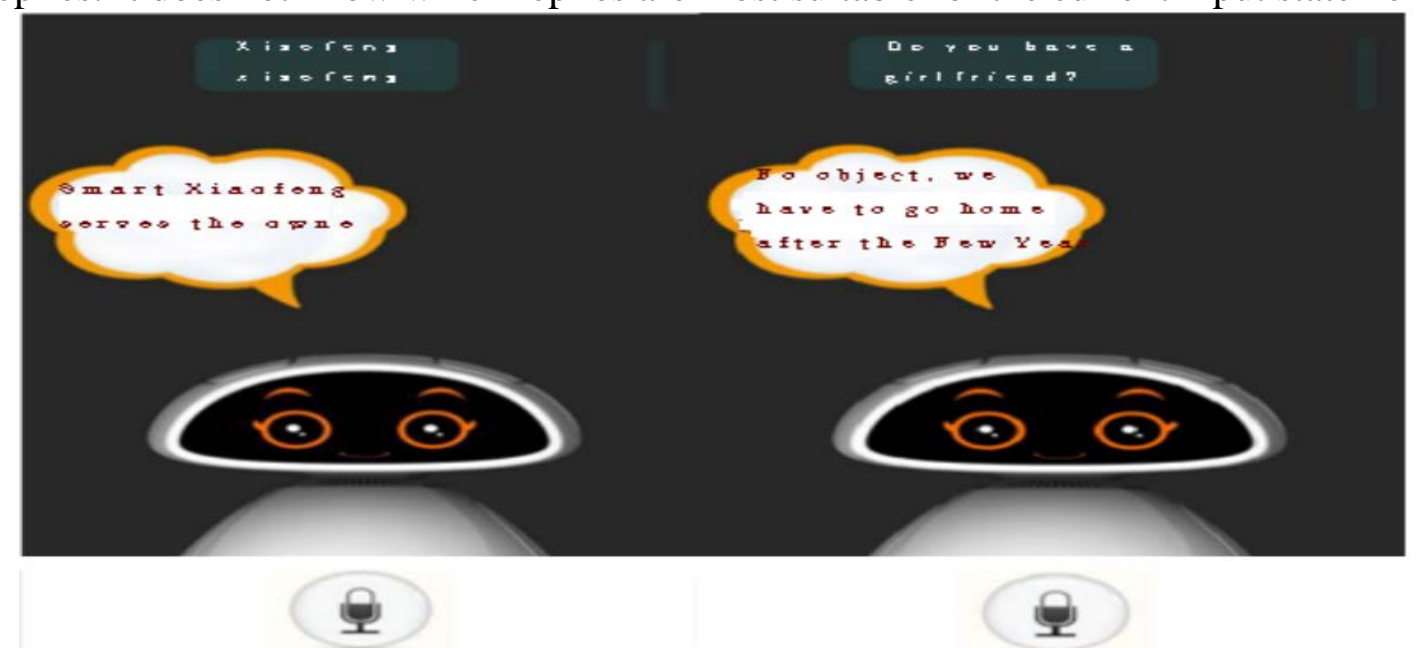

Fig. 2 smart Xiaofeng main interface and rejection judgment

\section{Results Analysis}

After opening the application program, Smart Xiaofeng will present the service interface to the eyes of the experiencer, and the smart robot will give a visual impact. Based on the above test data, the following responses were made to the "Smart Xiaofeng" response:

Accurate positioning. The user said: "Hi, Xiaofeng. How is the weather today? Is there any rain?"

"Smart Xiaofeng": "Wednesday, May 30, 2018, April 23, 2005, April 23, $23^{\circ} \mathrm{C}$, sunny, $17^{\circ} \mathrm{C} \sim 30^{\circ} \mathrm{C}$, Humidity: 35\%, Wind direction: South-East winds, Class 2 UV: The weakest." "Smart Xiaofeng" is accurate. Locate the location of the user and read out the weather in the detailed area.

Memory learning. "Come to a Jacky Cheung song" followed by saying: "Another one."

"Smart Xiaofeng" can remember just what the user stated, and will sing Zhang Xueyou's "Kissing" again. "Smart Xiaofeng" will move his smart mouth, cute and playful.

Speech dictation. User input: "Li Bai's quiet night thinking" and "Smart Xiaofeng" immediately followed by a "quiet night thinking Li Bai's bed before the moonlight, suspected of frost on the ground. Looking up at the moon and moon, head down to think of home." 
Rejection judgment. "What is your private life?", "Do you have a girlfriend?", "You are a bad person."

"Smart Xiaofeng" has a conscious euphemism for refusing to say the sentence, the language is slightly playful, "No object, good, New Year's home to go again."

\section{Function Introduction}

The implementation function contains five functional modules. The speech recognition module is responsible for receiving the user's speech input and converting it into a text form for processing by the natural language understanding module. The dialogue management module is responsible for coordinating the invocation of each module and maintaining the current dialogue state, selecting a specific reply mode and processing it by the natural language generating module.

User Intention Recognition: The user intention includes explicit intention and implicit intention. The intention of the display usually corresponds to a specific requirement. For example, if the user inputs "I want to reserve a standard room", it clearly indicates the intention of the room reservation.

User emotion recognition: The user's emotions also include both explicit and implicit. For example, if the user enters "I'm speeching today", it clearly indicates the emotion of joy, and "the exam has just passed the exam" is not easy. Determine the user's emotions.

Memory ability: The robot can memorize the questions that the user had talked about before, such as: "The user has entered the day before to take two courses, and in the later chat, he mentioned that he took a class today and asked the robot user a total of After a few exams, the robot will reply two."

Precise positioning: Accurately locate and output accurate information based on the information provided by the user. For example: "When the user enters today's weather conditions, the chat robot will accurately locate the user's location and read out the weather forecast for this day."

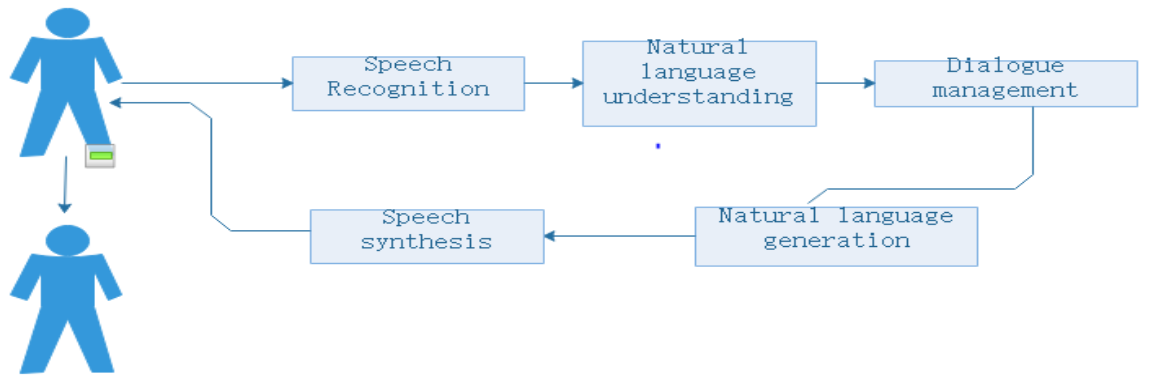

Fig. 3 System Implementation Framework

\section{Features}

Intelligent chat robot features introduction:

Learning ability. This is the most fundamental and most difficult criterion to raise. A robot that can grow automatically but has a small amount of data is obviously more powerful than one that cannot grow automatically but has a large amount of data. The essence of the robot is to help users and reduce the amount of labor in various fields. If a robot needs to manually enter all the knowledge, this will itself increase the burden on the user. Although "Smart Little Peak" is With a small amount of data, but its learning ability is super strong, it can grow automatically and store users' questions in the process of interacting with users, so as to continuously store data and expand data volume.

Big data screening capability. After having the self-learning ability, the robot is a knowledge of whether to receive a complete knowledge or a selective study, which is a criterion for further evaluating the intelligence level of the robot. If the robot can only collect knowledge, the learning ability is incomplete, and the robot should have more or less ability to filter. When the user enters the same question before and after, "Smart Xiaofeng" can intelligently reply to different forms.

Self-upgrade capability. After "Smart Xiaofeng" has done self-learning according to the designer's data structure and algorithm and has a certain level of knowledge screening capability, 
self-upgrade capability will become the next bastion. The essence of learning ability is to structure the input data into the data organization structure of this robot according to the designer's algorithm. After "getting all types of input data according to the initial algorithm", it was found that when such rules cannot adapt to a certain type of knowledge organization or domain knowledge, designers should consider how to make robots try to adjust the algorithm rules and data organization structure.

Accurate positioning. When the user sends a request: “Today's weather conditions, or the user's location.” The Xiaofeng Summit uses a high-precision satellite positioning system and has an automatic map generation function. Reply to "Tai'an weather forecast, your location is Tai'an."

Human-computer interaction. The main functions of the "Intelligent Xiaofeng" under the intelligent Q\&A scenario include answering the factual questions raised by users in the form of natural language and the problems that require calculation and logical reasoning in order to directly meet the user's information needs and assist the user in making decisions.

\section{Conclusions}

This article introduces the overview of intelligent robots and their research background, and elaborates the basic principles and key technologies of intelligent robots. The research on big data analysis and deep learning has led to the development of chat robots. Chatting robots will gradually replace APP as the next generation service portal for the Internet. The research of this paper has broadened the features of the robot on the basis of the existing technology. The interface is also different from other chat robots. It has unique features. The main interface has a small robot and can be very natural. Smart Xiaofeng can accurately read out the description.

Smart Xiaofeng has functions such as accurate positioning, memory learning, speech dictation, speech recognition, rejection judgment, user emotion recognition, user intention recognition, big data screening, and psychological counseling. Insufficient functions will be repaired in an upgraded version developed later, optimization and perfection.

\section{Acknowledgments}

This research has been partially supported by the national Spark Program project (No.:2014GA740055), science and technology development project of Tai'an city (No.: 20140630-6), science and technology plan project of Shandong province (No.: J14LN68), Taishan university's young teachers' research fund project (No.: QN-01-201702). This research has been partially supported by Shandong province undergraduate colleges and universities Research project on teaching reform; Shandong province soft science research plan; Ministry of education cooperative education comprehensive reform project. We like to express our appreciation for the valuable suggestions from the referee and the editor of this journal which significantly improved the quality of the presented paper.

\section{References}

[1] Xiao Kecong. Research and implementation of intelligent chat robot with emotion recognition ability, Beijing University of Posts and Telecommunications, China 2017. (In Chinese)

[2] Cheng Jie. Smart Chat Robot: Dialogue in the Future Science and Technology Economics Journal, Vol. 02(2018):p.14-15. (In Chinese)

[3] Tan M.H. and Ranganath S: Communications and Signal Processing, Vol.3, 2003, p.1335-1340. (In Chinese) 